\title{
MODELING ARTERIAL WALL DRUG CONCENTRATIONS FOLLOWING THE INSERTION OF A DRUG-ELUTING STENT
}

\author{
SEAN MCGINTY ${ }^{\dagger}$, SEAN MCKEE ${ }^{\dagger}$, ROGER M WADSWORTH, AND CHRISTOPHER \\ MCCORMICK $\S$
}

\begin{abstract}
A mathematical model of a drug-eluting stent is proposed. The model considers a polymer region, containing the drug initially, and a porous region consisting of smooth muscle cells embedded in an extracellular matrix. An analytical solution is obtained for the drug concentration both in the target cells and the interstitial region of the tissue in terms of the drug release concentration at the interface between the polymer and the tissue. When the polymer region and the tissue region are considered as a coupled system it can be shown, under certain assumptions, that the drug release concentration satisfies a Volterra integral equation which must be solved numerically in general. The drug concentrations, both in the cellular and extracellular regions, are then determined from the solution of this integral equation and used in deriving the mass of drug in the cells and extracellular space.
\end{abstract}

Key words. drug-eluting stent, atherosclerosis, Laplace transforms, branch points, analytical solution, Volterra integral equation.

AMS subject classifications. 92C50, 35E99, 76R99, 76S99, 44A10

1. Introduction. Coronary heart disease (CHD) is the main cause of death in developed countries [32] and accounts for $18 \%$ of all deaths in the United States annually [26]. CHD is characterized by a blockage or occlusion of one or more of the arteries which supply blood to the heart muscle. This is due to atherosclerosis, a complex progressive inflammatory disease [25], which leads to the build up of fatty plaque material near the inner surface of the arterial wall [29]. If left untreated, this leads to episodes of chest pain (angina). Ultimately, the atherosclerotic plaque is vulnerable to rupture, leading to the formation of a blood clot which blocks the artery, causing a heart attack. Until relatively recently, by-pass surgery was required. However, in the majority of cases this has now been replaced by inserting a small metallic cage called a stent into the occluded artery to maintain blood flow. When a stent is implanted into an artery, the endothelium is severely damaged. The consequent inflammatory response and excessive proliferation and migration of smooth muscle cells leads to the development of in-stent restenosis (ISR), a re-occlusion of the artery which is a significant limitation of bare metal stents. The introduction of drug-eluting stents (DES) significantly reduced the occurrence of ISR, by releasing a drug to inhibit smooth muscle cell proliferation. However, their use has been associated with incomplete healing of the artery and substantial efforts are now dedicated towards the development of enhanced DESs. Photographs of typical stent designs are provided below (see Figure 1.1) while Figure 1.2 displays the stent, in situ in the coronary artery.

An important aspect in the performance of any DES is the drug release profile. Although a number of animal models are recommended for preclinical safety and efficacy evaluation of these devices [38], incomplete understanding of the factors

\footnotetext{
$\dagger$ Department of Mathematics and Statistics, University of Strathclyde, 26 Richmond Street, Glasgow, G1 1XH, UK. (s.mcginty@strath.ac.uk) (Corresponding author.)

$¥$ The author is deceased. Former address: Strathclyde Institute of Pharmacy and Biomedical Sciences, University of Strathclyde, Glasgow G4 0NR, UK.

§Biomedical Engineering, 106 Rottenrow, University of Strathclyde, Glasgow G4 0NW, UK.

IStrathclyde Institute of Pharmacy and Biomedical Sciences, University of Strathclyde, Glasgow G4 0NR, UK.
} 

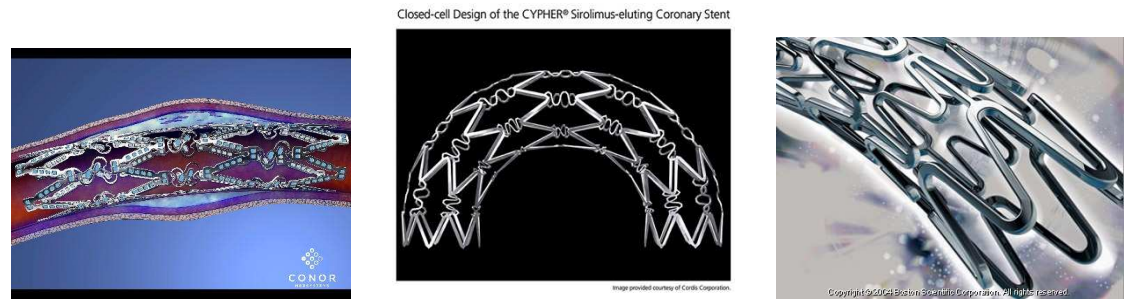

FIG. 1.1. A selection of stent designs.

governing drug release and distribution following stenting currently limits the optimisation of such drug release profiles. Several authors have focussed specifically on the drug release problem. Zhao et al. [45] presented an analytical solution of a cylindrical diffusion model to describe the experimental drug release of everolimus from a Dynalink-E polymer coated stent. They demonstrated that the release could be controlled by varying the coating thickness and diffusion coefficient and found that their model solution could be fitted to both in vitro and in vivo data simply by varying the diffusion coefficient. Formaggia et al. [15] considered a dissolution-diffusion model which also incorporated polymer degradation while Prabhu et al. [36] focused specifically on the degradation and release of everolimus from a polylactic stent coating and validated their compartmentalized model using in vitro data. A number of other contributions in the literature have attempted to combine mathematical modeling with experimental validation. Balakrishnan et al. [2] utilised computational fluid dynamics and two dimensional transient convection-diffusion to make predictions of drug elution which were then verified using empirical data from stented porcine arteries. They found that arterial uptake was only maximized when the rates of drug release and absorptions matched. Rossi et al. [37] modeled a bioresorbable DES based on detailed constitutive equations and taking into account the main physical and chemical mechanisms involved in coating degradation, drug release and restenosis inhibition. Their results were verified against selected in vitro and in vivo data available in the literature. Most recently, Tzafriri et al. [42] developed a mathematical framework of arterial drug distribution and receptor binding following stent elution. Their model predicted that tissue content linearly tracked stent elution rate; this prediction was validated in porcine coronary artery sirolimus-eluting polymer coated stent implants. In an attempt to make some gains in modeling this complex problem, Seidlitz et al. [39] utilised a vessel-simulating flow through cell to examine release from DES in vitro. This tool allowed for the examination of diffusion depth and the distribution in the arterial wall.

Several computational approaches to drug release from stents have been employed. Lovich and Edelman [28], Constantini et al. [10], and McGinty et al. [30] numerically studied a one-dimensional model. Two-dimensional models were computed by Hwang et al. [20], Zunino [47] and Grassi et al. [16]. A three-dimensional model in a simplified geometry was studied by Hose et al. [19] while, Vairo et al. [43] considered a multidomain approach. Zunino et al. [48] presented three-dimensional numerical models of stent expansion and release into the blood flow and tissue while the effect of luminal flow on arterial deposition is considered in [23] and [24]. Karner and Perktold [21] used the finite element method to calculate the transport processes across the lumen, the intima and the media, coupled with the flux across the endothelium and the internal elastic lamina which they modeled mathematically using the Kedem- 
Katchalsky equations. Delfour et al. [14], on the other hand, focused on the effect of the number of struts and the ratio between the coated area, and attempted to optimize the effect of the dose. Mongrain et al. [31] numerically investigated the effect of diffusion coefficient and struts apposition on drug accumulation in the arterial wall. More recently, Tambaca et al. [41] presented a mathematical model for the study of the mechanical properties of endovascular stents in their expanded state. Realising the sheer complexity of modeling the whole stent-tissue system, D'Angelo et al. [13] employed model reduction strategies to simplify the computations. This involved a combination of lumped parameter models to account for drug release, a one dimensional model to handle the complex stent pattern and a three dimensional model for drug transfer in the artery.

Despite the aforementioned numerical advances, there is still a lack of analytical solutions in the literature, especially for the coupled stent tissue system. Two interesting papers by Pontrelli and de Monte ([33], [34]) considered a similar model to the more general (but still one-dimensional) models of McGinty et al. [30]. Their model is a two layer model (drug concentration in both the polymer and the tissue regions) and, through the Kedem-Katchalsky equations, allows for a topcoat. They obtained an elegant analytical solution through separation of variables and used this solution to show the effect of filtration velocity, drug metabolism and the amount (i.e. the mass) of drug in the tissue. Pontrelli and de Monte have subsequently extended their work to consider a multi-layer model [35]. Mathematical models which admit analytical solutions, such as the one considered in this paper, have a real part to play in addressing this complex problem.

This paper develops a mathematical model for drug release from polymer coated DES and the subsequent uptake into the arterial wall. Having written down a model which makes certain simplifying assumptions, the strategy is the following. First we assume that we know the drug release concentration at the interface between the polymer and the tissue, say $g(t)$. This allows us to obtain a general analytical solution for any $g(t)$ both for the drug concentration in the extracellular matrix and the smooth muscle cells themselves. This analytical solution involves the inversion of a Laplace transform with three branch points. We then examine the concentration in the polymer and observe that, if $g(t)$ were known, the problem would be overdetermined. Indeed we can write down two independent well-posed problems, both of which admit analytical solutions. However, the arbitrary function $g(t)$, representing drug release, must be such that the two solutions are identical. Hence, by equating these two solutions, it is possible to derive a Volterra integral equation for $g(t)$. We then proceed to solve this integral equation for $g(t)$ and utilise this in computing the mass of drug in the cells and extracellular space. Thus we have an alternative approach to the two-layered model of Pontrelli and de Monte, that is capable of modeling the drug concentration within the target smooth muscle cells, which is what the clinicians are principally interested in. This is a novel approach to solving this problem and, moreover, can be regarded as a generic mathematical tool for solving these types of diffusion systems.

2. The structure of the arterial wall. The arterial wall is a heterogeneous structure, consisting of three distinct layers: the intima, the media and the adventitia [44] - see Figure 2.1. The intima is the innermost region closest to the lumen. The main constituent of the intima is the endothelial layer of cells, known as the endothelium. This layer is crucial to the control of the normal function of the artery, through its mediation of relaxation and contraction and via its control of smooth muscle cell 


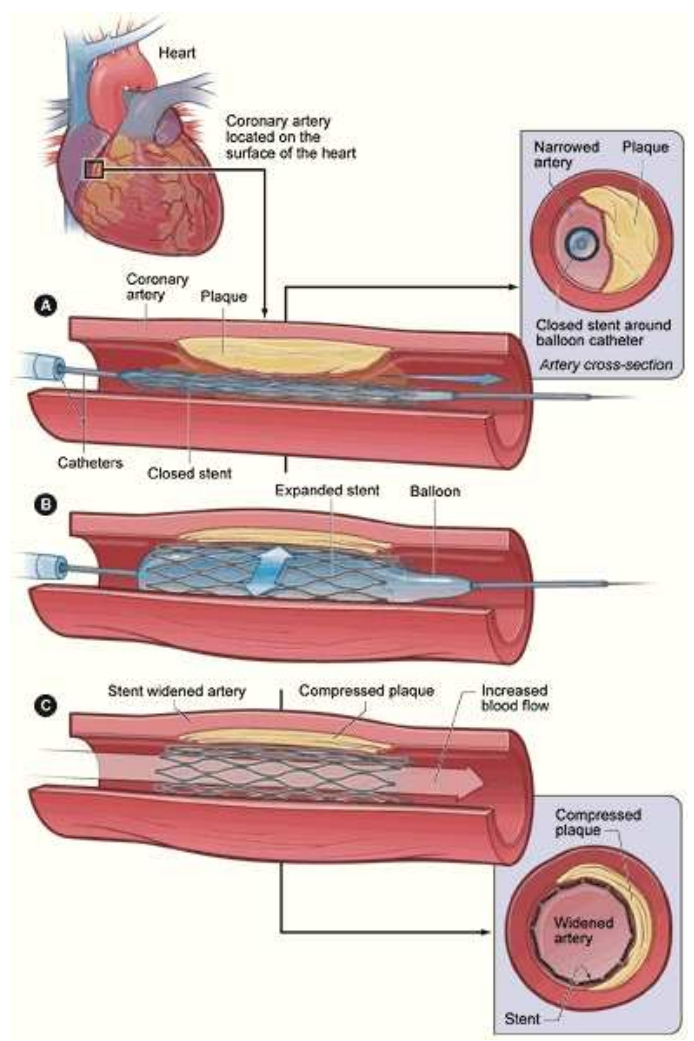

FIG. 1.2. The illustration shows a cross-section of a coronary artery with plaque buildup. The coronary artery is located on the surface of the heart. In A we see the deflated balloon catheter inserted into the narrowed coronary artery. In $B$, the balloon is inflated, compressing the plaque and restoring the size of the artery. Finally, C shows the widened artery. (Credit: US National Heart Lung and Blood institute)

proliferation within the underlying media layer. The internal elastic lamina (a fenestrated layer of elastic tissue) forms the outermost part of the intima. The next layer is the media (middle) region containing smooth muscle cells, collagen and elastin. Finally, the outermost layer of the arterial wall is the adventitia, which is separated from the media by the external elastic lamina. Essentially, the adventitia tethers the artery to perivascular tissue, and contains cells known as fibroblasts. There is also the presence of a network of small blood vessels, termed vasa vasorum, which act as a blood supply to the adventitia and provide a clearance mechanism for drugs released into the artery wall.

3. The mathematical model. To obtain a tractable mathematical model we shall make certain assumptions about the structure of the arterial wall. The intima, when it is devoid of the endothelium, has a similar structure to the media region and for this reason we shall not include the intima as a separate region in the model. Secondly, the adventitia is omitted since there is some evidence in the literature [30] that the adventitia does not have a large effect on the cellular drug concentration in the media region. The media consists of two phases: one of smooth muscle cells, collagen and elastin surrounded by an interstitial (or extracellular) region (the second phase). We treat the media as a porous region, where the porosity, $\phi \in(0,1)$, is 


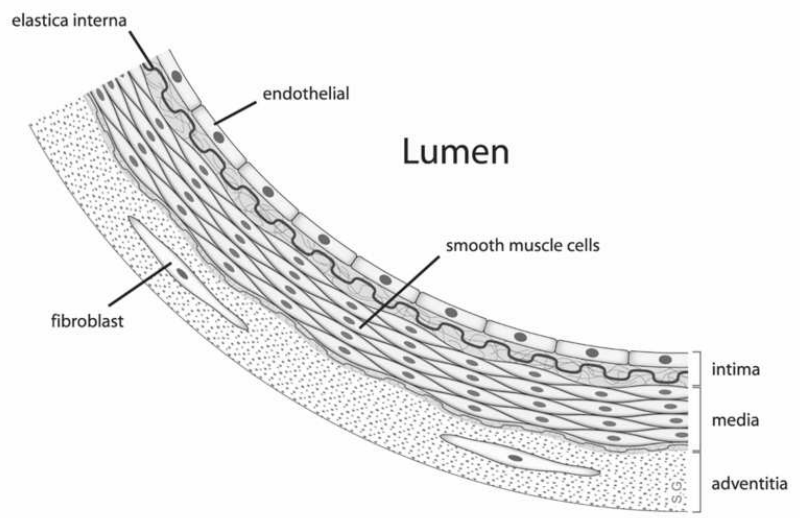

FIG. 2.1. The structure of the arterial wall.

defined as the ratio of the volume of interstitial space to the total volume. The drug is known to have a partition coefficient, $K$, that is, the equilibrium ratio of concentrations of a compound in two different phases, and we regard the drug as interacting with the cells, but not diffusing within them. It is well accepted (see, for example, [3]) that there is a transmural flow of plasma across the intima and through the media causing the drug to convect. We shall assume the velocity of this flow, $v$, is constant. The purpose of including the drug within the stent is to target and hence inhibit the smooth muscle cells which constitute the neointima. Figure 3.1 provides a diagrammatic sketch of the simplified physiology of the artery wall. Of course, these assumptions are somewhat gross, but they do have the advantage that they allow some progress towards an analytical solution rather than a purely numerical one.

We consider a stent coated with a thin layer (of thickness $L$ ) of polymer containing a drug and embedded into the arterial wall (of thickness $L_{1}$ ) as schematically illustrated in Figure 3.1. We introduce $c_{1}(x, t)$ and $c_{2}(x, t)$ which denote, respectively, the concentration of the drug in the interstitial region (of the media) and the concentration of the drug within the cells. The drug concentration in the polymer, $c(x, t)$, is assumed to satisfy a diffusion equation with diffusion coefficient $D$ while the transport of drug through the media is governed by an advection-diffusion equation where the transmural velocity of the plasma is denoted by $v$ and $D_{1}$ denotes the drug diffusion coefficient through the interstitial region. We include an uptake equation, with drug uptake rate constant $\alpha$, to describe the uptake of drug into the cells within the tissue. The mathematical model is

$$
\begin{gathered}
\frac{\partial c}{\partial t}=D \frac{\partial^{2} c}{\partial x^{2}}, \quad x \in(-L, 0), \quad t>0, \\
D \frac{\partial c}{\partial x}=0, \quad x=-L, \quad t>0, \\
c=c_{0}, \quad x \in[-L, 0], \quad t=0, \\
D \frac{\partial c}{\partial x}=D_{1} \frac{\partial c_{1}}{\partial x}-v c_{1}, \quad x=0, t>0,
\end{gathered}
$$




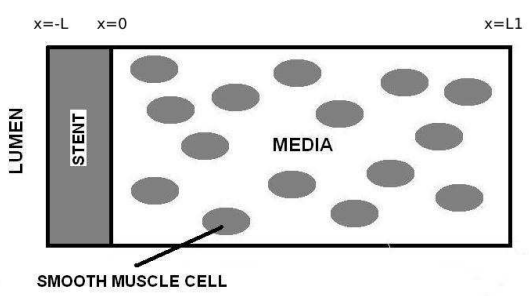

FIG. 3.1. Simplified geometry displaying stent impinged against media region containing smooth muscle cells and extracellular space.

$$
\begin{gathered}
-D \frac{\partial c}{\partial x}=P\left(c-c_{1}\right), \quad x=0, t>0 \\
\phi \frac{\partial c_{1}}{\partial t}+v \frac{\partial c_{1}}{\partial x}=D_{1} \frac{\partial^{2} c_{1}}{\partial x^{2}}-\alpha\left(c_{1}-c_{2} / K\right), \quad x \in\left(0, L_{1}\right), t>0 \\
c_{1}, c_{2} \text { bounded, } \quad x \in\left[0, L_{1}\right], t \in[0, \infty), \\
c_{1}=c_{2}=0, \quad x \in\left[0, L_{1}\right], t=0 \\
(1-\phi) \frac{\partial c_{2}}{\partial t}=\alpha\left(c_{1}-c_{2} / K\right), \quad x \in\left(0, L_{1}\right), t>0 .
\end{gathered}
$$

This model assumes that the drug in the polymer is in a single phase which is permitted to freely diffuse. This is appropriate where the initial concentration of drug in the polymer is below solubility, in which case the dissolution of drug can be regarded as instantaneous [8]. If the initial concentration of drug in the polymer is above solubility, then the drug may exist in two forms, crystalline and dissolved, with only dissolved drug free to diffuse. An approximate solution to the problem of drug release into an infinite sink for this case is provided by Higuchi [17]. Generalizations of Higuchi's work were considered by Biscari et al. [6] and Cohen and Erneux [9]. However, in some DES systems, it may well be the case that the initial concentration of the drug in the polymer is above solubility. In this case, if diffusion is the governing step in the release process then our assumption is still valid [40]. In this paper we focus on the polymer coated Cypher sirolimus-eluting stent (see Section 6 for a description). We have conducted experiments within our laboratory which examine in-vitro drug release from the Cypher stent. Figure 3.2 displays a comparison between the experimentally measured cumulative percentage of drug released, and the widely published solution of (3.1-3.3) along with the condition $c=0$ at $x=0$ (see, for example Crank [12]). The good agreement between the model and the experiments serves to demonstrate that diffusion is the dominant mechanism of release, at least for this particular stent. The best-fitting value of $D$ based on a least squares analysis was found to be of the order $10^{-16} \mathrm{~m}^{2} \mathrm{~s}^{-1}$.

We assume that the polymer rests against the bare metal of the stent and consequently there is zero flux at $x=-L$. For simplicity the initial concentration within the polymer is assumed to be some constant $c_{0}$. At the polymer/tissue interface the total flux is assumed to be continuous, and a top-coat on the polymer is allowed for 


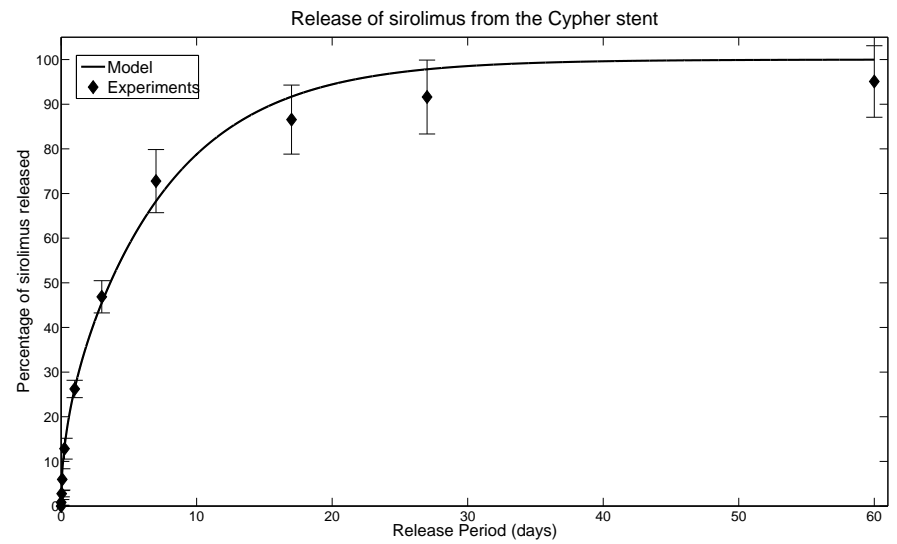

FIG. 3.2. Comparison between in-vitro experimental data and diffusion based model of sirolimus release from the Cypher stent. Briefly, the experiments consisted of placing four Cypher DESs in separate sealed glass vials containing physiological release medium (phosphate buffered saline:ethanol (90:10)). The experiments were carried out at $37^{\circ} \mathrm{C}$. At several time points up to 60 days, each stent was removed and placed in a separate vial containing fresh release medium, with the mass of drug in the original solution subsequently quantified using $U V$-spectroscopy.

via the condition (3.5), where, here, $P$ denotes a parameter with units $m s^{-1}$ (see e.g. [33]). Equation (3.6) governs the movement of the drug in the interstitial region of the media. Note that the 'sink' term represents the removal of drug to the cells. Some existing models consider binding sites within the tissue (see e.g. [42], [7]). It is certainly true that the drug will bind to binding sites in the tissue and in the cells ([42], [5], [27]), although the strength of affinity will likely vary substantially with the particular drug under consideration and further, it is not clear how the density of the binding sites may be easily determined. The uptake of drug into smooth muscle cells has been measured experimentally (see, for example [46]). The model considered here does not account for binding sites in the extracellular matrix, but instead considers cells within the tissue absorbing and releasing drug as the concentration in the extracellular region changes. This idea is supported by the following authors ([1], [18]). It is worth stating that no boundary condition has been stipulated at the media/adventitia interface. An argument could be made for imposing continuity of the relative diffusive and convective fluxes across the interface, or simply that the extracellular concentration falls to zero by the time the drug reaches the adventitia region. These boundary conditions, and others, have been considered in the literature (for example [30], [47], [33]). However, in order to be able to obtain an analytical solution, we do not state a boundary condition at $x=L_{1}$, but instead impose the condition that the concentration of drug in the extracellular region and the cells must remain bounded for all $x$ and $t$.

Since the system is linear, one obvious approach would be to solve directly using Laplace transforms. However, it is readily seen that this approach leads to a Laplace transform which is simply impractical to invert. Further, any attempt to proceed with the inversion would necessarily involve extremely complicated transcendental equations that must be solved for the roots. Thus we consider the following novel approach to solving this problem. Consider a simpler hypothetical problem where we assume that we know $c_{1}(0, t)$ in terms of a general function of $t$, say $g(t)$. This will 
allow us to obtain the concentrations for the drug both in the cells and the interstitial region (in terms of $g(t)$ ) and, as we shall see, reduce the problem of finding $g(t)$ to that of solving a Volterra integral equation.

We shall introduce the following non-dimensional variables:

$$
t^{\prime}=\left(D_{1} / L_{1}^{2}\right) t, x^{\prime}=x / L_{1}, c^{\prime}=c / c_{0}, c_{1}^{\prime}=c_{1} / c_{0}, c_{2}^{\prime}=c_{2} / c_{0},
$$

so that the above model becomes (all primes have been omitted for reasons of clarity):

$$
\begin{aligned}
& \frac{\partial c}{\partial t}=\delta \frac{\partial^{2} c}{\partial x^{2}}, x \in(-\ell, 0), t>0 \\
& \frac{\partial c}{\partial x}=0, x=-\ell, t>0 \\
& c=1, x \in[-\ell, 0], t=0 \\
& \delta \frac{\partial c}{\partial x}=\frac{\partial c_{1}}{\partial x}-P e c_{1}, x=0, t>0 \\
& -\frac{\partial c}{\partial x}=\tilde{P}\left(c-c_{1}\right), x=0, t>0 \\
& \phi \frac{\partial c_{1}}{\partial t}+P e \frac{\partial c_{1}}{\partial x}=\frac{\partial^{2} c_{1}}{\partial x^{2}}-D a\left(c_{1}-c_{2} / K\right), x \in(0,1), t>0, \\
& c_{1}, c_{2} \text { bounded, } x \in[0,1], t>0, \\
& c_{1}=c_{2}=0, x \in[0,1], t=0, \\
& (1-\phi) \frac{\partial c_{2}}{\partial t}=D a\left(c_{1}-c_{2} / K\right), x \in(0,1), t>0
\end{aligned}
$$

Here $\delta=D / D_{1}, \ell=L / L_{1}, \tilde{P}=L_{1} P / D, P e=L_{1} v / D_{1}$ (Peclet number) and $D a=L_{1}^{2} \alpha / D_{1}$ (Damkholer number).

4. An analytical solution. Consider the problem consisting of $(3.6)^{\prime}-(3.9)^{\prime}$ together with $c_{1}(0, t)=g(t)$. We shall now apply Laplace transforms to equations $(3.6)^{\prime}$ and $(3.9)^{\prime}$. We shall then discover, upon applying the complex inversion formula, that the integrand has three branch points, requiring a modification of the usual Bromwich contour and leading ultimately to an analytical solution in terms of the unknown drug release concentration, $g(t)$.

4.1. Solution in Laplace transform space. Rearranging $(3.9)^{\prime}$ provides

$$
\frac{\partial c_{2}}{\partial t}(x, t)+\frac{\gamma}{K} c_{2}(x, t)=\gamma c_{1}(x, t)
$$

where

$$
\gamma=\frac{D a}{1-\phi}
$$

Solving (4.1) subject to the initial condition gives

$$
c_{2}(x, t)=\gamma \int_{0}^{t} e^{-\gamma\left(t-t^{\prime}\right) / K} c_{1}\left(x, t^{\prime}\right) d t^{\prime}
$$

After substituting (4.2) into (3.6)', we obtain

$$
\phi \frac{\partial c_{1}}{\partial t}(x, t)+P e \frac{\partial c_{1}}{\partial x}(x, t)=\frac{\partial^{2} c_{1}}{\partial x^{2}}(x, t)-D a\left(c_{1}(x, t)-\frac{\gamma}{K} \int_{0}^{t} e^{-\gamma\left(t-t^{\prime}\right) / K} c_{1}\left(x, t^{\prime}\right) d t^{\prime}\right) .
$$


Define the Laplace transform of $c_{i}(x, t)(i=1,2)$ with respect to $t$ :

$$
\bar{c}_{i}(x, s)=\int_{0}^{\infty} e^{-s t} c_{i}(x, t) d t .
$$

Now, taking Laplace transforms of (4.3) yields, after making use of the initial condition and rearranging,

$$
\frac{d^{2} \overline{c_{1}}}{d x^{2}}(x, s)-P e \frac{d \overline{c_{1}}}{d x}(x, s)-\Gamma(s) \overline{c_{1}}(x, s)=0,
$$

where

$$
\Gamma(s)=\frac{\phi K s\left(s+\frac{\gamma}{K}+\frac{D a}{\phi}\right)}{K s+\gamma} .
$$

Solving (4.4) subject to $c_{1}(0, t)=g(t)$ and the boundedness of $c_{1}(x, t)$, we obtain

$$
\overline{c_{1}}(x, s)=\bar{g}(s) \exp \left\{\frac{x P e}{2}\right\} \exp \left\{-\frac{x}{2} \sqrt{P e^{2}+4 \Gamma(s)}\right\},
$$

where $\bar{g}(s)=\int_{0}^{\infty} e^{-s t} g(t) d t$. Using the definition of $\Gamma(s)$ from (4.5), it is possible to re-write (4.6) in a more transparent form which clearly displays the dependence on $s$ :

$$
\overline{c_{1}}(x, s)=\bar{g}(s) \exp \left\{\frac{x P e}{2}\right\} \exp \left\{-x \sqrt{\phi} \sqrt{\frac{\left(s+s_{1}\right)\left(s+s_{2}\right)}{s+s_{3}}}\right\},
$$

where

$$
\begin{aligned}
2 s_{1,2} & =\frac{\gamma}{K}+\frac{D a}{\phi}+\frac{P e^{2}}{4 \phi} \mp \sqrt{\left(\frac{\gamma}{K}+\frac{D a}{\phi}+\frac{P e^{2}}{4 \phi}\right)^{2}-\frac{\gamma P e^{2}}{\phi K}}, \\
s_{3} & =\frac{\gamma}{K} .
\end{aligned}
$$

Finally, taking the Laplace transform of (4.2) (and using convolution) allows us to write down the solution of $c_{2}$ in Laplace transform space:

$$
\begin{aligned}
\overline{c_{2}}(x, s) & =\frac{\gamma}{s+s_{3}} \overline{c_{1}}(x, s) \\
& =\frac{\gamma \bar{g}(s)}{\left(s+s_{3}\right)} \exp \left\{\frac{x P e}{2}\right\} \exp \left\{-x \sqrt{\phi} \sqrt{\frac{\left(s+s_{1}\right)\left(s+s_{2}\right)}{s+s_{3}}}\right\} .
\end{aligned}
$$

4.2. Solution via complex inversion formula. It will be convenient first to determine the inverse of the following Laplace transform

$$
\bar{f}(s)=\frac{\exp \left\{-x \sqrt{\phi} \sqrt{\frac{\left(s+s_{1}\right)\left(s+s_{2}\right)}{\left(s+s_{3}\right)}}\right\}}{s},
$$




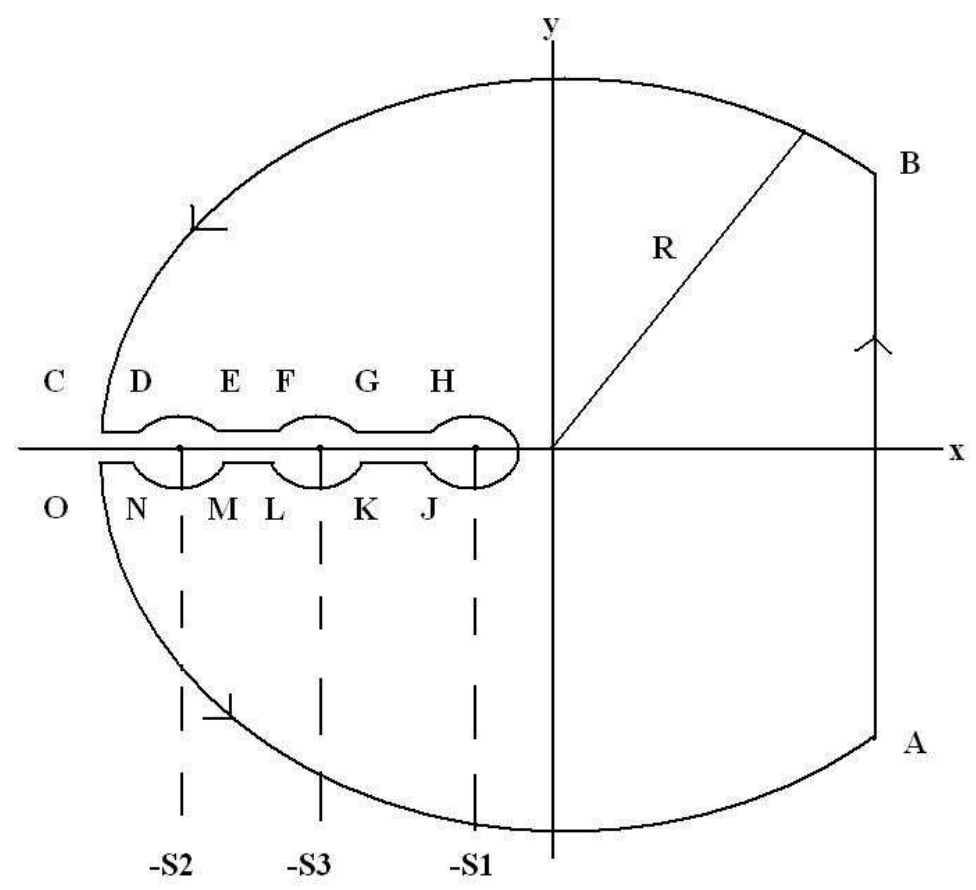

Fig. 4.1. Modified Bromwich Contour. Note that the circles centered on $-s_{1},-s_{3}$ and $-s_{2}$ have radii $\epsilon_{1}, \epsilon_{3}$ and $\epsilon_{2}$, respectively. $A, B, . . N, O$ are defined in the appendix.

that is, by the complex inversion formula, we require

$$
f(t)=L^{-1}[\bar{f}(s)]=\frac{1}{2 \pi i} \int_{\beta-i \infty}^{\beta+i \infty} \frac{\exp \left\{s t-x \sqrt{\phi} \sqrt{\frac{\left(s+s_{1}\right)\left(s+s_{2}\right)}{\left(s+s_{3}\right)}}\right\}}{s} d s .
$$

To evaluate (4.12) we consider the modified Bromwich contour in Figure 4.1. We observe that the integrand of (4.12) has a simple pole at $s=0$ and three branch points at $-s_{1},-s_{3}$ and $-s_{2}$. We shall later demonstrate that the typical values of the parameters characterizing the model are such that $0<s_{1}<s_{3}<s_{2}$. Thus a branch cut has been made along the negative real axis. The details of the inversion are provided in the appendix. The solution $f(t)$ turns out to be

$$
f(t)=\exp \left\{-x \sqrt{\frac{\phi s_{1} s_{2}}{s_{3}}}\right\}-\frac{1}{\pi}\left(I_{1}+\tilde{I}_{1}\right)
$$

where

$$
I_{1}=\int_{s_{2}}^{\infty} \frac{e^{-u t} \sin (a(u) x)}{u} d u, \quad \tilde{I}_{1}=\int_{s_{1}}^{s_{3}} \frac{e^{-u t} \sin (a(u) x)}{u} d u
$$

with

$$
a(u)=\sqrt{\frac{\phi\left(u-s_{1}\right)\left(u-s_{2}\right)}{\left(u-s_{3}\right)}} .
$$


Note that we can rewrite (4.7) as

$$
\overline{c_{1}}(x, s)=\exp \left(\frac{x P e}{2}\right) s \bar{g}(s) \bar{f}(s) .
$$

An application of the convolution theorem to (4.16) and integration by parts results in

$$
c_{1}(x, t)=\exp \left(\frac{x P e}{2}\right)\left[g(t) \exp \left\{-x \sqrt{\frac{\phi s_{1} s_{2}}{s_{3}}}\right\}-\frac{g(t)\left(I_{2}+\tilde{I}_{2}\right)}{\pi}+\frac{I_{3}+\tilde{I}_{3}}{\pi}\right]
$$

where

$$
I_{2}=\int_{s_{2}}^{\infty} \frac{\sin (a(u) x)}{u} d u, \quad \tilde{I}_{2}=\int_{s_{1}}^{s_{3}} \frac{\sin (a(u) x)}{u} d u
$$

and

$I_{3}=\int_{s_{2}}^{\infty} \int_{0}^{t} g\left(t^{\prime}\right) e^{-u\left(t-t^{\prime}\right)} \sin (a(u) x) d t^{\prime} d u, \tilde{I}_{3}=\int_{s_{1}}^{s_{3}} \int_{0}^{t} g\left(t^{\prime}\right) e^{-u\left(t-t^{\prime}\right)} \sin (a(u) x) d t^{\prime} d u$.

A further application of the Convolution Theorem to (4.10) then yields

$$
c_{2}(x, t)=\gamma \exp \left(\frac{x P e}{2}\right)\left[\exp \left\{-x \sqrt{\frac{\phi s_{1} s_{2}}{s_{3}}}\right\} I_{4}-\frac{I_{5}+\tilde{I}_{5}}{\pi}+\frac{I_{6}+\tilde{I}_{6}}{\pi}\right]
$$

where

$$
\begin{gathered}
I_{4}=\int_{0}^{t} g\left(t^{\prime}\right) e^{-s_{3}\left(t-t^{\prime}\right)} d t^{\prime} \\
I_{5}=\int_{s_{2}}^{\infty} \int_{0}^{t} g\left(t^{\prime}\right) e^{-s_{3}\left(t-t^{\prime}\right)} \frac{\sin (a(u) x)}{u} d t^{\prime} d u, \tilde{I}_{5}=\int_{s_{1}}^{s_{3}} \int_{0}^{t} g\left(t^{\prime}\right) e^{-s_{3}\left(t-t^{\prime}\right)} \frac{\sin (a(u) x)}{u} d t^{\prime} d u
\end{gathered}
$$

and

$$
\begin{aligned}
& I_{6}=\int_{s_{2}}^{\infty} \int_{0}^{t} \int_{0}^{\tau} g\left(t^{\prime}\right) e^{-u\left(\tau-t^{\prime}\right)} e^{-s_{3}(t-\tau)} \sin (a(u) x) d t^{\prime} d \tau d u \\
& \tilde{I}_{6}=\int_{s_{1}}^{s_{3}} \int_{0}^{t} \int_{0}^{\tau} g\left(t^{\prime}\right) e^{-u\left(\tau-t^{\prime}\right)} e^{-s_{3}(t-\tau)} \sin (a(u) x) d t^{\prime} d \tau d u
\end{aligned}
$$

5. A Volterra integral equation. We have obtained $c_{1}(x, t)$ and $c_{2}(x, t)$ in terms of the arbitrary function $g(t)$. The object now is to determine $g(t)$. Consider the problem

$$
\begin{aligned}
\frac{\partial c}{\partial t} & =\delta \frac{\partial^{2} c}{\partial x^{2}}, x \in(-\ell, 0), t>0, \\
\frac{\partial c}{\partial x} & =0, x=-\ell, t>0, \\
c & =1, x \in[-\ell, 0], t=0, \\
\delta \frac{\partial c}{\partial x} & =\frac{\partial c_{1}}{\partial x}-P e c_{1}, x=0, t>0, \\
-\frac{\partial c}{\partial x} & =\tilde{P}\left(c-c_{1}\right), x=0, t>0,
\end{aligned}
$$


where we intend to replace $c_{1}(0, t)$ by $g(t)$. If we were able to determine $\partial c_{1}(0, t) / \partial x$ (and hence $\partial c(0, t) / \partial x)$ then (5.1)-(5.5) would be over-determined. This "overdeterminedness" could then be used to find an expression for $g(t)$. However, it is not difficult to see that $\partial c_{1}(0, t) / \partial x$ is not defined: this is a direct consequence of the discontinuity in the boundary and initial condition at $x=0$ and $t=0$ of the model defined by (3.1)-(3.9). A form of regularization is required. We have chosen to approximate $\partial c_{1}(0, t) / \partial x$ by utilising the solution of the corresponding problem of purely diffusion in a semi-infinite composite region. To be precise here, we mean the solution obtained by solving $(3.1)^{\prime}$ to $(3.7)^{\prime}$ where $[0,1]$ has been replaced by $[0, \infty)$, $\phi=1$ and $P e$ and $D a$ have been taken to be zero. It can be readily shown that, in this case,

$$
\begin{aligned}
\frac{\partial c_{1}(0, t)}{\partial x} & =\frac{\tilde{P} \sqrt{\delta}}{\pi} \int_{0}^{\infty} \frac{B \sin (l \sqrt{u / \delta}) \exp (-u t)}{\sqrt{u}\left(A^{2}+B^{2}\right)} d u \\
& =j(t), \text { say, }
\end{aligned}
$$

where

$$
A=\tilde{P} \cos (l \sqrt{u / \delta})-(\sqrt{u / \delta}) \sin (l \sqrt{u / \delta})
$$

and

$$
B=\tilde{P} \sqrt{\delta} \sin (l \sqrt{u / \delta})
$$

Thus, we regularize by replacing (5.4) by

$$
\delta \frac{\partial c}{\partial x}=j(t)-P e c_{1}, \quad x=0, t>0 .
$$

Consider the further transformation of the independent variables

$$
t^{\prime}=\left(\delta / \ell^{2}\right) t, \quad x^{\prime}=\left(\frac{x+\ell}{\ell}\right),
$$

so that equations (5.1)-(5.3), (5.5) and (5.6) become

$$
\begin{aligned}
\frac{\partial c}{\partial t} & =\frac{\partial^{2} c}{\partial x^{2}}, \quad x \in(0,1), t>0, \\
\frac{\partial c}{\partial x} & =0, \quad x=0, t>0, \\
c & =1, \quad x \in[0,1], t=0, \\
\frac{\partial c}{\partial x} & =\frac{\ell}{\delta} j(t)-\frac{\ell P_{e}}{\delta} g(t), \quad x=1, t>0, \\
-\frac{\partial c}{\partial x} & =P^{*}(c-g(t)), \quad x=1, t>0,
\end{aligned}
$$

where $P^{*}=\ell \tilde{P}=L P / D$. Again, the primes have been omitted for clarity. We are now in a position to write down two problems and their associated solutions. 


\section{Problem 1}

$$
\begin{array}{rlrl}
\frac{\partial c}{\partial t} & =\frac{\partial^{2} c}{\partial x^{2}}, & x \in(0,1), t>0, \\
\frac{\partial c}{\partial x} & =0, & x & =0, t>0, \\
c & =1, & x \in[0,1], t=0, \\
\frac{\partial c}{\partial x} & =\frac{\ell}{\delta} j(t)-\frac{\ell P_{e}}{\delta} g(t), & x=1, t>0 .
\end{array}
$$

The following solution may be obtained from either an application of Laplace transforms or separation of variables with Duhamel's theorem:

$$
c(x, t)=1-\frac{\ell}{\delta} \int_{0}^{t}\left(P_{e} g(\tau)-j(\tau)\right)\left[1+2 \sum_{n=1}^{\infty}(-1)^{n} \exp \left(-n^{2} \pi^{2}(t-\tau)\right) \cos (n \pi x)\right] d \tau .
$$

\section{Problem 2}

$$
\begin{aligned}
\frac{\partial c}{\partial t} & =\frac{\partial^{2} c}{\partial x^{2}}, & x \in(0,1), t>0, \\
\frac{\partial c}{\partial x} & =0, & x=0, t>0, \\
c & =1, & x \in[0,1], t=0, \\
-\frac{\partial c}{\partial c} & =P^{*}(c-g(t)), & x=1, t>0 .
\end{aligned}
$$

In a similar manner the following solution may be obtained:

$$
\begin{aligned}
c(x, t)= & 2 P^{*} \sum_{n=0}^{\infty} \frac{\exp \left(-\xi_{n}^{2} t\right) \cos \left(\xi_{n} x\right)}{\xi_{n}\left[\left(1+P^{*}\right) \sin \xi_{n}+\xi_{n} \cos \xi_{n}\right]} \\
& +2 P^{*} \int_{0}^{t} g(\tau) \sum_{n=0}^{\infty} \frac{\xi_{n} \exp \left(-\xi^{2}(t-\tau)\right) \cos \left(\xi_{n} x\right)}{\left[\left(1+P^{*}\right) \sin \xi_{n}+\xi_{n} \cos \xi_{n}\right]} d \tau
\end{aligned}
$$

where $\xi_{n}, n=0,1,2, \ldots$ are the countably infinite roots of

$$
\xi \tan \xi=P^{*} .
$$

The two solutions (5.17) and (5.22) must be identical, i.e. valid for all values of $x \in[0,1]$ and $t>0$. We shall select to equate the two integrals (over $x$ ) of (5.17) and (5.22). The physical significance of this is that we are effectively equating the respective expressions for the mass of drug on the stent. Thus

$$
\begin{aligned}
& 2 P^{*} \sum_{n=0}^{\infty} \frac{\exp \left(-\xi_{n}^{2} t\right)}{\xi_{n}\left[\left(1+P^{*}\right) \sin \xi_{n}+\xi_{n} \cos \xi_{n}\right]} \int_{0}^{1} \cos \left(\xi_{n} x\right) d x \\
+ & 2 P^{*} \int_{0}^{t} g(\tau) \sum_{n=0}^{\infty} \frac{\xi_{n} \exp \left(-\xi_{n}^{2}(t-\tau)\right)}{\left[\left(1+P^{*}\right) \sin \xi_{n}+\xi_{n} \cos \xi_{n}\right]} \int_{0}^{1} \cos \left(\xi_{n} x\right) d x d \tau \\
= & 1-\frac{\ell}{\delta} \int_{0}^{t}\left(P_{e} g(\tau)-j(\tau)\right)\left[1+2 \sum_{n=1}^{\infty}(-1)^{n} \exp \left(-n^{2} \pi^{2}(t-\tau)\right) \int_{0}^{1} \cos (n \pi x) d x\right] d \tau
\end{aligned}
$$


and so

$$
\begin{aligned}
& 2 P^{*} \sum_{n=0}^{\infty} \frac{\exp \left(-\xi_{n}^{2} t\right) \sin \xi_{n}}{\xi_{n}^{2}\left[\left(1+P^{*}\right) \sin \xi_{n}+\xi_{n} \cos \xi_{n}\right]} \\
+ & 2 P^{*} \int_{0}^{t} g(\tau) \sum_{n=0}^{\infty} \frac{\exp \left(-\xi_{n}^{2}(t-\tau)\right) \sin \xi_{n}}{\left[\left(1+P^{*}\right) \sin \xi_{n}+\xi_{n} \cos \xi_{n}\right]} d \tau \\
= & 1-\frac{\ell}{\delta} \int_{0}^{t}\left(P_{e} g(\tau)-j(\tau)\right) d \tau .
\end{aligned}
$$

Differentiating with respect to $t$ gives

$$
\begin{aligned}
& -2 P^{*} \sum_{n=0}^{\infty} \frac{\exp \left(-\xi_{n}^{2} t\right) \sin \xi_{n}}{\left[\left(1+P^{*}\right) \sin \xi_{n}+\xi_{n} \cos \xi_{n}\right]}+2 P^{*}\left(g(t) \sum_{n=0}^{\infty} \frac{\sin \xi_{n}}{\left[\left(1+P^{*}\right) \sin \xi_{n}+\xi_{n} \cos \xi_{n}\right]}\right. \\
& \left.(5.25) \quad-\int_{0}^{t} g(t) \sum_{n=0}^{\infty} \frac{\xi_{n}^{2} \exp \left(-\xi_{n}^{2}(t-\tau)\right) \sin \xi_{n}}{\left[\left(1+P^{*}\right) \sin \xi_{n}+\xi_{n} \cos \xi_{n}\right]} d \tau\right) \\
& \quad=-\frac{\ell}{\delta}\left(P_{e} g(t)-j(t)\right) .
\end{aligned}
$$

Re-arranging yields

$$
\begin{aligned}
& \left\{2 P^{*} \sum_{n=0}^{\infty} \frac{\sin \xi_{n}}{\left[\left(1+P^{*}\right) \sin \xi_{n}+\xi_{n} \cos \xi_{n}\right]}+\frac{\ell P_{e}}{\delta}\right\} g(t) \\
= & \int_{0}^{t}\left\{2 P^{*} \sum_{n=0}^{\infty} \frac{\xi_{n}^{2} \exp \left(-\xi_{n}^{2}(t-\tau)\right) \sin \xi_{n}}{\left[\left(1+P^{*}\right) \sin \xi_{n}+\xi_{n} \cos \xi_{n}\right]}\right\} g(\tau) d \tau \\
+ & 2 P^{*} \sum_{n=0}^{\infty} \frac{\exp \left(-\xi_{n}^{2} t\right) \sin \xi_{n}}{\left[\left(1+P^{*}\right) \sin \xi_{n}+\xi_{n} \cos \xi_{n}\right]}+\frac{\ell}{\delta} j(t)
\end{aligned}
$$

Solving this integral equation for $g(t)$ allows us to determine $c_{1}(x, t)$ and $c_{2}(x, t)$ through the analytical solutions obtained in Section 4. The concentration of drug within the polymer may then be obtained either from (5.17) or (5.22).

6. Parameter values. A common difficulty when modeling physiological processes is in obtaining estimates of the model parameters. Experimentation is often prohibitively expensive or simply not possible in vivo and it is therefore usual to draw data from different studies in the literature. We refer to [30], where an extensive literature search was performed to obtain estimates of the various parameters associated with drug elution from stents into arterial tissue. In this paper we will consider the non-erodible polymer coated Cypher sirolimus-eluting stent. We have chosen this particular stent system since it contains a drug-filled polymer coating and the mechanism of release is generally accepted to be diffusion. Thus this stent is well suited to our modeling assumptions. The Cypher stent coating is a blend of poly-ethyleneco-vinyl acetate (PEVA), poly-n-butyl methacrylate (PBMA) and the drug sirolimus. The coating is applied on a poly-o-chloro-p-xylylene (parylene-C) treated stainless 


\begin{tabular}{|c|c|c|}
\hline Parameter & Symbol & Value \\
\hline \hline Media Porosity & $\phi$ & 0.61 \\
\hline Media Thickness & $L_{1}$ & $4.5 \times 10^{-4} \mathrm{~m}$ \\
\hline Media Diffusion Coefficient & $D_{1}$ & $2.5 \times 10^{-10} \mathrm{~m}^{2} \mathrm{~s}^{-1}$ \\
\hline Drug Uptake Rate Constant & $\alpha$ & $2 \times 10^{-5} \mathrm{~s}^{-1}$ \\
\hline Partition Coefficient & $K$ & 15 \\
\hline Transmural Velocity & $v$ & $5.8 \times 10^{-8} \mathrm{~ms}^{-1}$ \\
\hline Polymer Diffusion Coefficient & $D$ & $10^{-16} \mathrm{~m}^{2} \mathrm{~s}^{-1}$ \\
\hline Permeability of Topcoat & $P$ & $10^{-8} \mathrm{~ms}^{-1}$ \\
\hline Polymer Thickness & $L$ & $1.26 \times 10^{-5} \mathrm{~m}$ \\
\hline
\end{tabular}

Table of parameter values based on McGinty et al. [30], Tzafriri et al. [42] and Pontrelli \& de Monte [33] [34].

steel stent. The manufacture of the Cypher consists of applying a basecoat solution containing PEVA, PBMA and the drug. An inactive topcoat and toulene spray is then applied. As a result of the mixing and drying process, drug is actually transported to the topcoat layer so that the drug-free topcoat is never actually realised [4]. Most of the newer generation stents also make use of limus compounds [22]. To consider different compounds in our model we would simply require measurements of the drug-dependent parameters. The value of media porosity is taken directly from [30]. The polymer thickness is taken from [11] and the diffusion coefficient of sirolimus in the Cypher stent has been measured in our laboratory to be of order $10^{-16} \mathrm{~m}^{2} \mathrm{~s}^{-1}$, while the values of the media diffusion coefficient of sirolimus, media thickness and transmural velocity have been taken from [42]. The value of the parameter $P$ has been taken from [33] whilst the values of the drug uptake constant and partition coefficient of sirolimus have been estimated based on [30]. Using the parameter values in Table 6.1 , we find that $s_{1}=3.7010 \times 10^{-4}, s_{2}=0.0334, s_{3}=0.0028$ to four decimal places, satisfying $0<s_{1}<s_{3}<s_{2}$.

7. Solution of the integral equation. Consider the discretization $\left\{t_{m}=\right.$ $m h, h=T / M, m=0,1, \ldots M\}$ and the associated vector $\left(g_{0}, g_{1}, \ldots, g_{m}\right)$ approximating $\left(g(0), g\left(t_{1}\right), \ldots, g\left(t_{m}\right)\right)$. We employ an explicit Euler-type method

$$
\begin{aligned}
& \left\{2 P^{*} \sum_{n=0}^{\infty} \frac{\sin \xi_{n}}{\left[\left(1+P^{*}\right) \sin \xi_{n}+\xi_{n} \cos \xi_{n}\right]}+\frac{\ell P_{e}}{\delta}\right\} g_{m} \\
= & h \sum_{j=0}^{m-1}\left\{2 P^{*} \sum_{n=0}^{\infty} \frac{\xi_{n}^{2} \exp \left(-\xi_{n}^{2}(m-j) h\right) \sin \xi_{n}}{\left[\left(1+P^{*}\right) \sin \xi_{n}+\xi_{n} \cos \xi_{n}\right]}\right\} g_{j} \\
& +2 P^{*} \sum_{n=0}^{\infty} \frac{\exp \left(-\xi_{n}^{2} m h\right) \sin \xi_{n}}{\left[\left(1+P^{*}\right) \sin \xi_{n}+\xi_{n} \cos \xi_{n}\right]}+\frac{\ell}{\delta} W_{m},
\end{aligned}
$$

with

$$
g_{0}=g(0)=\frac{2 P^{*} \Psi(\boldsymbol{\xi})+\ell W_{0} / \delta}{2 P^{*} \Psi(\boldsymbol{\xi})+\frac{\ell P e}{\delta}},
$$

where

$$
\Psi(\boldsymbol{\xi})=\sum_{n=0}^{\infty} \frac{\sin \xi_{n}}{\left[\left(1+P^{*}\right) \sin \xi_{n}+\xi_{n} \cos \xi_{n}\right]} .
$$


Before the discrete equation (7.1) can be solved, the roots of $\xi \tan \xi=P^{*}$ are required. These have been obtained using a bisection approach. The infinite sums in (7.1) were

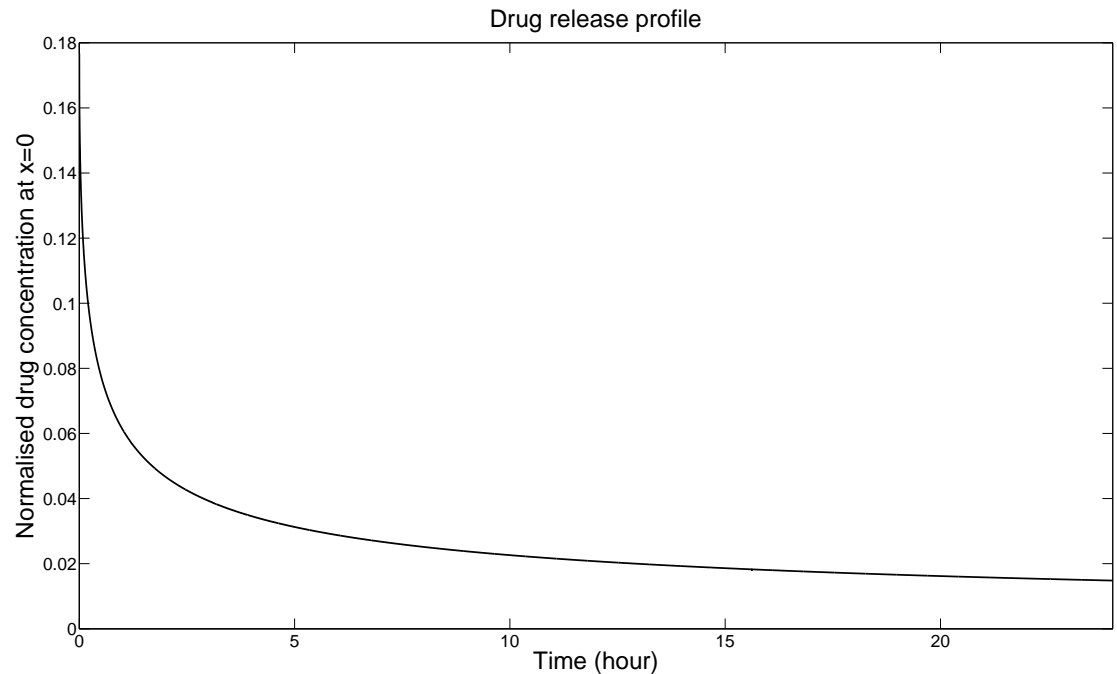

FIG. 7.1. $g(t)=c_{1}(0, t)$ over the first day as calculated from (5.26).

truncated after the first 20 terms and then, using the calculated roots, the finite difference equation (7.1) was solved. Figure 7.1 displays $g(t)=c_{1}(0, t)$ over the first day.

Now that $g(t)$ has been obtained, we can utilise this $g(t)$ in solution (4.17) and (4.18) to obtain the concentration of drug in the extracellular and cellular regions and these are displayed in Figures 7.2 and 7.3.

8. Drug mass within the tissue. The total mass of drug in the media region at time $t$ is given by integrating the expressions for cellular and extracellular concentration over the length of the media. In non-dimensional terms, this equates to

$$
M(t)=\int_{0}^{1} \phi c_{1}(x, t) d x+\int_{0}^{1}(1-\phi) c_{2}(x, t) d x .
$$

Again, for convenience, the primes are suppressed.

Figure 8.1 displays the variation in mass of drug within the tissue over a period of 10 days following stent deployment, using high-order global adaptive quadrature to evaluate the integrals. We see that most of the mass is contained out-with the extracellular space, due to the high partition coefficient of the drug. We have made two further assumptions in producing these plots. Firstly, it is assumed that all of the drug is eluted from the stent, i.e. no drug is retained within the polymer. There is some evidence in the literature that for some drug-eluting stents, not all of the drug is eluted and in fact some of the drug remains trapped in the stent. An example of such a stent is the paclitaxel-eluting Taxus ${ }^{T M}$ stent which is also a polymer coated stent. Experiments in our laboratory have revealed that for the sirolimus coated Cypher stent at least $99 \%$ of the drug is eluted in vitro within the first three months, which justifies this assumption. The second assumption is that all of the drug released from 


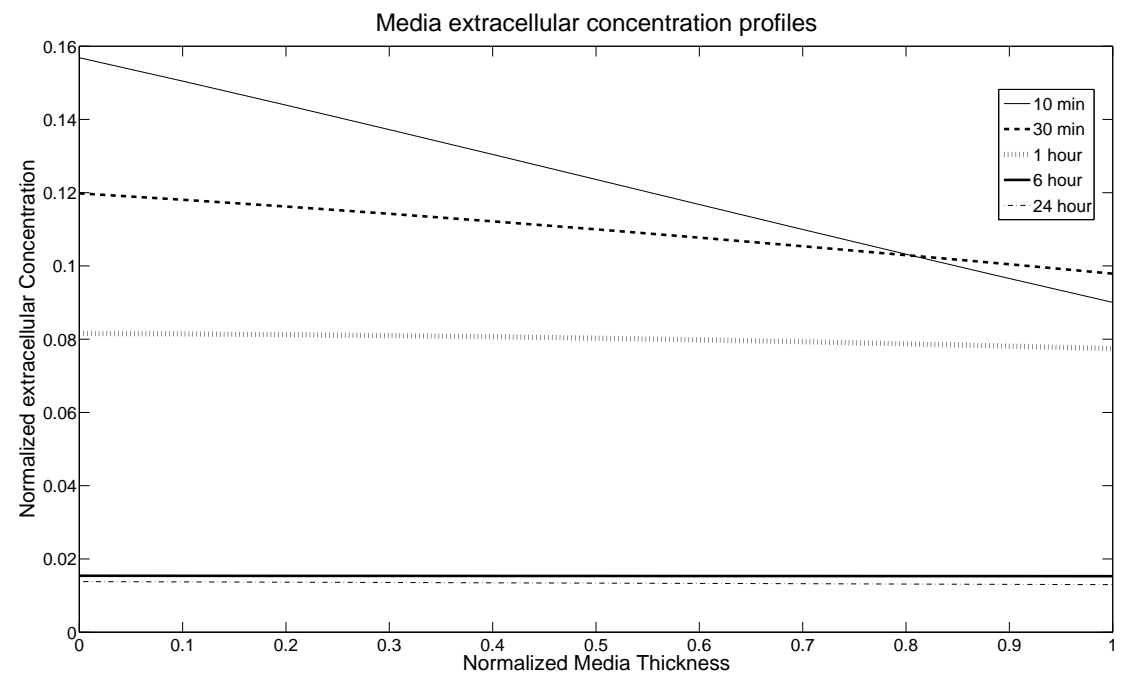

FIG. 7.2. Variation in extracellular concentration with time, subject to $g(t)$ obtained from the solution of the Volterra integral equation.

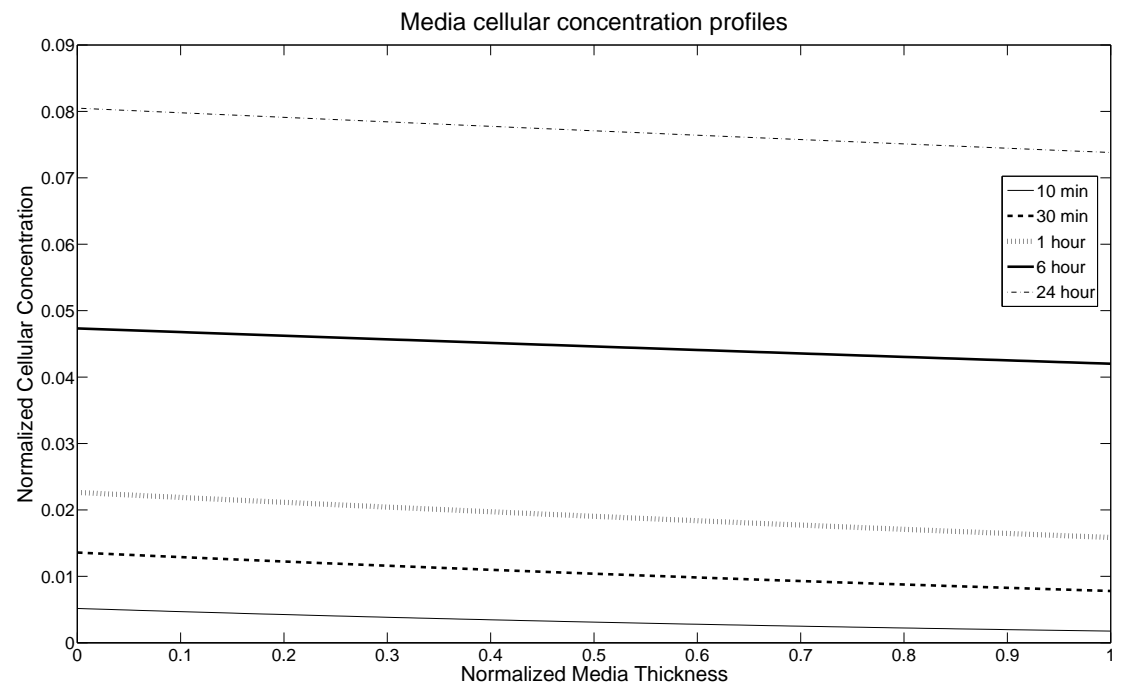

FIG. 7.3. Variation in cellular concentration with time, subject to $g(t)$ obtained from the solution of the Volterra integral equation.

the stent diffuses into the tissue. This assumption may be reasonable where the stent protrudes into the arterial wall.

9. Concluding remarks. In this paper we have developed analytical solutions for release of drug from a polymer coated stent into the arterial wall. By assuming initially that the drug release concentration at the polymer/tissue interface, $g(t)$, is known, we have been able to derive a Volterra integral equation, which allows us to 


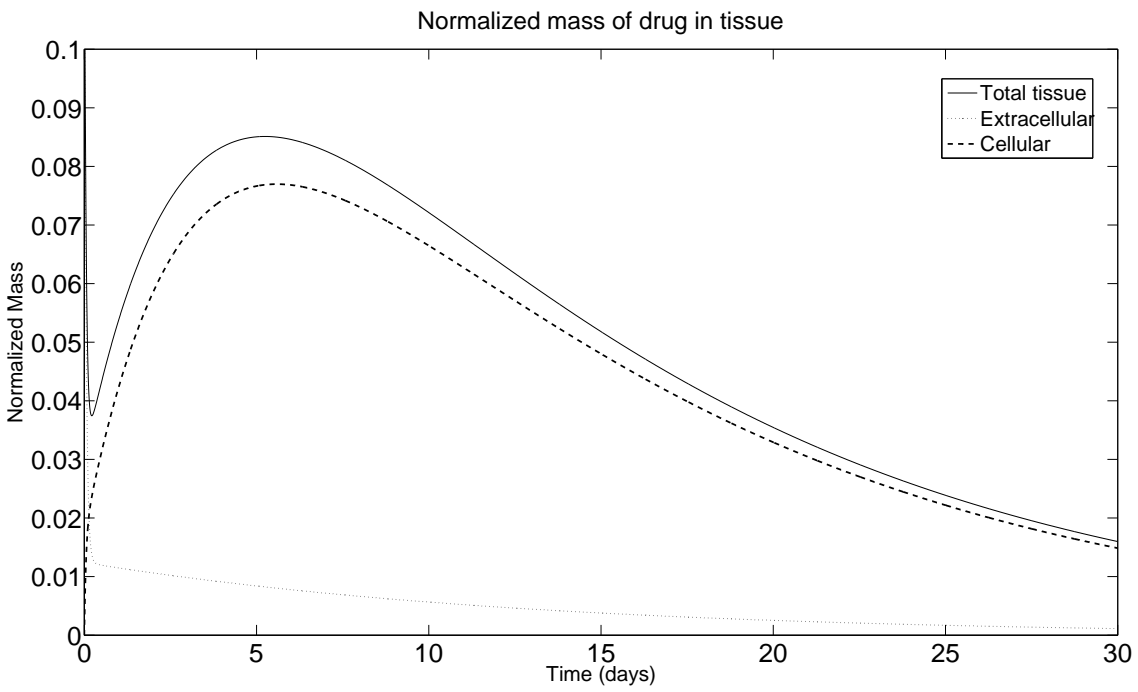

FIG. 8.1. Variation in mass of drug in the media with time, subject to $g(t)$ obtained from the solution of the Volterra integral equation

consider $g(t)$ as a variable of our model. Upon solving the integral equation for $g(t)$ we have been able to determine the drug concentration profiles in the extracellular and cellular regions of the arterial wall. Furthermore, we have calculated the mass of drug in each region over the period of release.

We feel it appropriate to comment on the limitations of our model. We have considered a one-dimensional model which, while making it difficult to generate quantitative results, nonetheless allow us to obtain qualitative results. However, primarily due to the regularization there is a negligible amount of mass loss. Furthermore, the model assumes that the main mechanism of release is diffusion. The problem of modeling arterial stents is very much an active field of research. The process of drug-release from the stent in vitro is still not fully understood, let alone the complex in vivo situation where flowing blood, pulsatility, wound healing, proliferation, migration of cells and complex uptake/binding no doubt all play some part. While we do not claim to have addressed all these issues, we believe that simple models which can admit analytical solutions, like the one presented here, have a part to play in addressing this complex problem.

10. Acknowledgements. We would like to thank the reviewers for their careful reading of the manuscript and for their suggestions and advice. We would also like to acknowledge the funding provided by EPSRC under grant number EP/J007242/1. The first author would also like to acknowledge the receipt of a Carnegie Scholarship. 
Appendix. Solution via complex inversion formula. Consider the following integral:

$$
\begin{aligned}
f(t) & =\frac{1}{2 \pi \mathrm{i}} \int_{\beta-\mathrm{i} \infty}^{\beta+\mathrm{i} \infty} \frac{\exp \left\{s t-x \sqrt{\phi} \sqrt{\frac{\left(s+s_{1}\right)\left(s+s_{2}\right)}{s+s_{3}}}\right\}}{s} d s . \\
& =\frac{1}{2 \pi \mathrm{i}} \int_{\beta-\mathrm{i} \infty}^{\beta+\mathrm{i} \infty} e^{s t} \bar{f}(s) d s .
\end{aligned}
$$

To evaluate (A.1), consider the modified Bromwich contour in Figure 4.1. Notice that the integrand of (A.1) has a simple pole at $s=0$ and three separate branch points at $-s_{1},-s_{3}$ and $-s_{2}$. For the parameter values considered, it is always the case that $0<s_{1}<s_{3}<s_{2}$. Thus a branch cut has been made along the negative real axis (see Figure 4.1).

Now,

$$
\begin{aligned}
& \frac{1}{2 \pi \mathrm{i}} \oint_{C} e^{s t} \bar{f}(s) d s \\
= & \frac{1}{2 \pi \mathrm{i}} \int_{A B} e^{s t} \bar{f}(s) d s+\frac{1}{2 \pi \mathrm{i}} \int_{B C} e^{s t} \bar{f}(s) d s+\frac{1}{2 \pi \mathrm{i}} \int_{C D} e^{s t} \bar{f}(s) d s \\
+ & \frac{1}{2 \pi \mathrm{i}} \int_{D E} e^{s t} \bar{f}(s) d s+\frac{1}{2 \pi \mathrm{i}} \int_{E F} e^{s t} \bar{f}(s) d s+\frac{1}{2 \pi \mathrm{i}} \int_{F G} e^{s t} \bar{f}(s) d s \\
+ & \frac{1}{2 \pi \mathrm{i}} \int_{G H} e^{s t} \bar{f}(s) d s+\frac{1}{2 \pi \mathrm{i}} \int_{H J} e^{s t} \bar{f}(s) d s+\frac{1}{2 \pi \mathrm{i}} \int_{J K} e^{s t} \bar{f}(s) d s \\
+ & \frac{1}{2 \pi \mathrm{i}} \int_{K L} e^{s t} \bar{f}(s) d s+\frac{1}{2 \pi \mathrm{i}} \int_{L M} e^{s t} \bar{f}(s) d s+\frac{1}{2 \pi \mathrm{i}} \int_{M N} e^{s t} \bar{f}(s) d s \\
+ & \frac{1}{2 \pi \mathrm{i}} \int_{N O} e^{s t} \bar{f}(s) d s+\frac{1}{2 \pi \mathrm{i}} \int_{O A} e^{s t} \bar{f}(s) d s \\
= & \operatorname{Res}(s=0)
\end{aligned}
$$

so that there are fifteen integrals to consider. It can be readily shown that as $R \rightarrow \infty$, the integrals over $B C$ and $O A$ vanish.

Along $C D$, let $s=u e^{i \theta}, \theta=\pi, s+s_{1}=u_{1} e^{i \theta_{1}}, \theta_{1}=\pi, s+s_{2}=u_{2} e^{i \theta_{2}}, \theta_{2}=\pi$, $\overline{s+s}_{3}=u_{3} e^{i \theta_{3}}, \theta_{3}=\pi$, from $s=-R$ to $s=-s_{2}-\epsilon_{2}$ :

$$
\begin{aligned}
& \frac{1}{2 \pi i} \int_{C D} e^{s t} f(s) d s \\
= & \frac{1}{2 \pi i} \int_{-R}^{-s_{2}-\epsilon_{2}} e^{s t} f(s) d s \\
= & \frac{1}{2 \pi i} \int_{R}^{\epsilon_{2}+s_{2}} \frac{\exp \left\{-u t-x \sqrt{\phi} \sqrt{\frac{u_{1} e^{i \pi} u_{2} e^{i \pi}}{u_{3} e^{i \pi}}}\right\}}{-u}(-d u) \\
= & \frac{1}{2 \pi i} \int_{R}^{\epsilon_{2}+s_{2}} \frac{\exp \left\{-u t-i x \sqrt{\phi} \sqrt{\frac{u_{1} u_{2}}{u_{3}}}\right\}}{u} d u, \\
= & \frac{1}{2 \pi i} \int_{R}^{\epsilon_{2}+s_{2}} \frac{\exp \left\{-u t-i x \sqrt{\phi} \sqrt{\frac{\left(u-s_{1}\right)\left(u-s_{2}\right)}{u-s_{3}}}\right\}}{u} d u .
\end{aligned}
$$


Along $D E$, the point $-s_{2}$ is moved to the origin by writing $s=\epsilon_{2} e^{i \theta}-s_{2}$, $\overline{d s=i \epsilon_{2} e^{i \theta}} d \theta$. Thus

$$
\begin{aligned}
& \frac{1}{2 \pi i} \int_{D E} e^{s t} f(s) d s \\
= & \frac{\epsilon_{2}}{2 \pi} \int_{\pi}^{0} \frac{\exp \left\{\left(\epsilon_{2} e^{i \theta}-s_{2}\right) t-x \sqrt{\phi} \sqrt{\epsilon_{2}} e^{i \theta / 2} \sqrt{\frac{\epsilon_{2} e^{i \theta}+s_{1}-s_{2}}{\epsilon_{2} e^{i \theta}-s_{2}+s_{3}}}+i \theta\right\}}{\epsilon_{2} e^{i \theta}-s_{2}} d \theta \\
\rightarrow & 0 \text { as } \epsilon_{2} \rightarrow 0 .
\end{aligned}
$$

Along $E F$, let $s=u e^{i \theta}, \theta=\pi, s+s_{1}=u_{1} e^{i \theta_{1}}, \theta_{1}=\pi, s+s_{2}=u_{2} e^{i \theta_{2}}, \theta_{2}=0$, $\overline{s+s_{3}=u_{3}} e^{i \theta_{3}}, \theta_{3}=\pi$, from $s=-s_{2}+\epsilon_{2}$ to $s=-s_{3}-\epsilon_{3}$ :

$$
\begin{aligned}
& \frac{1}{2 \pi i} \int_{E F} e^{s t} f(s) d s \\
= & \frac{1}{2 \pi i} \int_{-s_{2}+\epsilon_{2}}^{-s_{3}-\epsilon_{3}} e^{s t} f(s) d s \\
= & \frac{1}{2 \pi i} \int_{s_{2}-\epsilon_{2}}^{s_{3}+\epsilon_{3}} \frac{\exp \left\{-u t-x \sqrt{\phi} \sqrt{\frac{\left(u-s_{1}\right)\left(s_{2}-u\right)}{u-s_{3}}}\right\}}{u} d u .
\end{aligned}
$$

Along $F G$, the point $-s_{3}$ is moved to the origin by writing $s=\epsilon_{3} e^{i \theta}-s_{3}$, $\overline{d s=i \epsilon_{3} e^{i \theta}} d \theta$. Thus

$$
\begin{aligned}
& \frac{1}{2 \pi i} \int_{F G} e^{s t} f(s) d s \\
= & \frac{\epsilon_{3}}{2 \pi} \int_{\pi}^{0} \frac{\exp \left\{-x \sqrt{\frac{\phi}{\epsilon_{3}}} e^{-i \theta / 2} \sqrt{\left(\epsilon_{3} e^{i \theta}+s_{1}-s_{3}\right)\left(\epsilon_{3} e^{i \theta}-s_{3}+s_{2}\right)}+i \theta\right\}}{\epsilon_{3} e^{i \theta}-s_{3}} d \theta \\
\rightarrow & 0 \text { as } \epsilon_{3} \rightarrow 0 .
\end{aligned}
$$

Along $G H$, let $s=u e^{i \theta}, \theta=\pi, s+s_{1}=u_{1} e^{i \theta_{1}}, \theta_{1}=\pi, s+s_{2}=u_{2} e^{i \theta_{2}}, \theta_{2}=0$, $\overline{s+s_{3}=u_{3}} e^{i \theta_{3}}, \theta_{3}=0$, from $s=-s_{3}+\epsilon_{3}$ to $s=-s_{1}-\epsilon_{1}$ :

$$
\begin{aligned}
& \frac{1}{2 \pi i} \int_{G H} e^{s t} f(s) d s \\
= & \frac{1}{2 \pi i} \int_{-s_{3}+\epsilon_{3}}^{-s_{1}-\epsilon_{1}} e^{s t} f(s) d s \\
= & \frac{1}{2 \pi i} \int_{s_{3}-\epsilon_{3}}^{s_{1}+\epsilon_{1}} \frac{\exp \left\{-u t-i x \sqrt{\phi} \sqrt{\left.\frac{\left(u-s_{1}\right)\left(s_{2}-u\right)}{s_{3}-u}\right\}}\right.}{u} d u .
\end{aligned}
$$

$\underline{\text { Along } H J}$, the point $-s_{1}$ is moved to the origin by writing $s=\epsilon_{1} e^{i \theta}-s_{1}$, 
$d s=i \epsilon_{1} e^{i \theta} d \theta$. Thus

$$
\begin{aligned}
& \frac{1}{2 \pi i} \int_{H J} e^{s t} f(s) d s \\
= & \frac{\epsilon_{1}}{2 \pi} \int_{\pi}^{-\pi} \frac{\exp \left\{\left(\epsilon_{1} e^{i \theta}-s_{1}\right) t-x \sqrt{\phi} \sqrt{\epsilon_{1}} e^{i \theta / 2} \sqrt{\frac{\epsilon_{1} e^{i \theta}-s_{1}+s_{2}}{\epsilon_{1} e^{i \theta}-s_{1}+s_{3}}}+i \theta\right\}}{\epsilon_{1} e^{i \theta}-s_{1}} d \theta \\
(\mathrm{A} .8) \rightarrow & 0 \text { as } \epsilon_{1} \rightarrow 0 .
\end{aligned}
$$

Along $J K$, let $s=u e^{i \theta}, \theta=-\pi, s+s_{1}=u_{1} e^{i \theta_{1}}, \theta_{1}=-\pi, s+s_{2}=u_{2} e^{i \theta_{2}}, \theta_{2}=0$, $\overline{s+s_{3}=u_{3}} e^{i \theta_{3}}, \theta_{3}=0$, from $s=-s_{1}-\epsilon_{1}$ to $s=-s_{3}+\epsilon_{3}$ :

$$
\begin{aligned}
& \frac{1}{2 \pi i} \int_{J K} e^{s t} f(s) d s \\
= & \frac{1}{2 \pi i} \int_{-s_{1}-\epsilon_{1}}^{-s_{3}+\epsilon_{3}} e^{s t} f(s) d s \\
= & \frac{1}{2 \pi i} \int_{s_{1}+\epsilon_{1}}^{s_{3}-\epsilon_{3}} \frac{\exp \left\{-u t+i x \sqrt{\phi} \sqrt{\frac{\left(u-s_{1}\right)\left(s_{2}-u\right)}{s_{3}-u}}\right\}}{u} d u .
\end{aligned}
$$

Along $K L$, the point $-s_{3}$ is moved to the origin by writing $s=\epsilon_{3} e^{i \theta}-s_{3}$, $\overline{d s=i \epsilon_{3} e^{i \theta}} d \theta$. Thus

$$
\begin{aligned}
& \frac{1}{2 \pi i} \int_{K L} e^{s t} f(s) d s \\
= & \frac{\epsilon_{3}}{2 \pi} \int_{0}^{-\pi} \frac{\exp \left\{-x \sqrt{\frac{\phi}{\epsilon_{3}}} e^{-i \theta / 2} \sqrt{\left(\epsilon_{3} e^{i \theta}+s_{1}-s_{3}\right)\left(\epsilon_{3} e^{i \theta}-s_{3}+s_{2}\right)}+i \theta\right.}{\epsilon_{3} e^{i \theta}-s_{3}} d \theta \\
\rightarrow & 0 \text { as } \epsilon_{3} \rightarrow 0 .
\end{aligned}
$$

Along $L M$, let $s=u e^{i \theta}, \theta=-\pi, s+s_{1}=u_{1} e^{i \theta_{1}}, \theta_{1}=-\pi, s+s_{2}=u_{2} e^{i \theta_{2}}, \theta_{2}=0$, $\overline{s+s_{3}=u_{3}} e^{i \theta_{3}}, \theta_{3}=-\pi$, from $s=-s_{3}-\epsilon_{3}$ to $s=-s_{2}+\epsilon_{2}$ :

$$
\begin{aligned}
& \frac{1}{2 \pi i} \int_{L M} e^{s t} f(s) d s \\
= & \frac{1}{2 \pi i} \int_{-s_{3}-\epsilon_{3}}^{-s_{2}+\epsilon_{2}} e^{s t} f(s) d s \\
= & \frac{1}{2 \pi i} \int_{s_{3}+\epsilon_{3}}^{s_{2}-\epsilon_{2}} \frac{\exp \left\{-u t-x \sqrt{\phi} \sqrt{\frac{\left(u-s_{1}\right)\left(s_{2}-u\right)}{u-s_{3}}}\right\}}{u} d u .
\end{aligned}
$$

Along $M N$, the point $-s_{2}$ is moved to the origin by writing $s=\epsilon_{2} e^{i \theta}-s_{2}$, $\overline{d s=i \epsilon_{2} e^{i \theta}} d \theta$. Thus

$$
\frac{1}{2 \pi i} \int_{M N} e^{s t} f(s) d s
$$




$$
\begin{aligned}
& =\frac{\epsilon_{2}}{2 \pi} \int_{0}^{-\pi} \frac{\exp \left\{\left(\epsilon_{2} e^{i \theta}-s_{2}\right) t-x \sqrt{\phi} \sqrt{\epsilon_{2}} e^{i \theta / 2} \sqrt{\frac{\epsilon_{2} e^{i \theta}+s_{1}-s_{2}}{\epsilon_{2} e^{i \theta}-s_{2}+s_{3}}}+i \theta\right\}}{\epsilon_{2} e^{i \theta}-s_{2}} d \theta \\
& \rightarrow 0 \text { as } \epsilon_{2} \rightarrow 0
\end{aligned}
$$

Along $N O$, let $s=u e^{i \theta}, \theta=-\pi, s+s_{1}=u_{1} e^{i \theta_{1}}, \theta_{1}=-\pi$, $\overline{s+s_{2}=u_{2}} e^{i \theta_{2}}, \theta_{2}=-\pi, s+s_{3}=u_{3} e^{i \theta_{3}}, \theta_{3}=-\pi$, from $s=-s_{2}-\epsilon_{2}$ to $s=-R$

$$
\begin{aligned}
& \frac{1}{2 \pi i} \int_{N O} e^{s t} f(s) d s \\
= & \frac{1}{2 \pi i} \int_{-s_{2}-\epsilon_{2}}^{-R} e^{s t} f(s) d s \\
= & \frac{1}{2 \pi i} \int_{s_{2}+\epsilon_{2}}^{R} \frac{\exp \left\{-u t+i x \sqrt{\phi} \sqrt{\frac{\left(u-s_{1}\right)\left(u-s_{2}\right)}{u-s_{3}}}\right\}}{u} d u .
\end{aligned}
$$

Now, the residue at the simple pole $s=0$ is

$$
\lim _{s \rightarrow 0} \frac{s \exp \left\{s t-x \sqrt{\phi} \sqrt{\frac{\left(s+s_{1}\right)\left(s+s_{2}\right)}{s+s_{3}}}\right\}}{s}=\exp \left\{-x \sqrt{\frac{\phi s_{1} s_{2}}{s_{3}}}\right\} .
$$

By the Residue Theorem,

$$
\frac{1}{2 \pi i} \oint_{C} e^{s t} f(s) d s=\exp \left\{-x \sqrt{\frac{\phi s_{1} s_{2}}{s_{3}}}\right\} .
$$

Hence, with the integrals along $B C, D E, F G, H J, K L, M N$ and $O A$ tending to zero in the limit, and with the integrals along $E F$ and $L M$ cancelling through addition, the only contributions are those from the integrals along $C D, G H, J K$ and $N O$. Thus, (A.2) reduces to

$$
\begin{aligned}
& \frac{1}{2 \pi i} \int_{A B} e^{s t} f(s) d s \\
&= \exp \left\{-x \sqrt{\frac{\phi s_{1} s_{2}}{s_{3}}}\right\} \\
&-\frac{1}{2 \pi i} \lim _{R \rightarrow \infty, \epsilon_{1,2,3} \rightarrow 0}\left\{\begin{array}{c}
\int_{C D} e^{s t} f(s) d s+\int_{G H} e^{s t} f(s) d s \\
+\int_{J K} e^{s t} f(s) d s+\int_{N O} e^{s t} f(s) d s
\end{array}\right\} \\
&= \exp \left\{-x \sqrt{\frac{\phi s_{1} s_{2}}{s_{3}}}\right\} \\
&-\frac{1}{\pi}\left\{\begin{array}{c}
\int_{s_{2}}^{\infty} \frac{e^{-u t}}{u} \sin \left(x \sqrt{\phi} \sqrt{\frac{\left(u-s_{1}\right)\left(u-s_{2}\right)}{u-s_{3}}}\right) d u \\
+\int_{s_{1}}^{s_{3}} \frac{e^{-u t}}{u}
\end{array}\right) d u \\
& \sin \left(x \sqrt{\phi} \sqrt{\frac{\left(u-s_{1}\right)\left(s_{2}-u\right)}{s_{3}-u}}\right) d u
\end{aligned}
$$

The solutions for $c_{1}$ and $c_{2}$ follow directly from (A.16) using convolution. 
[1] J. P. Abraham, J. M. Gorman, E. M. Sparrow, J. R. Stark and R. E. Kohler, A mass transfer model of temporal drug deposition in artery walls, Int. J. Heat Mass Trans., 58 (2013), pp. 632-638.

[2] B. Balakrishnan, J. F. Dooley, G. Kopia and E. R. Edelman, Intravascular drug release kinetics dictate arterial drug deposition, retention, and distribution, J. Controlled Release, 123(2) (2007), pp. 100-108.

[3] A. L. Baldwin, L. M. Wilson, I. Gradus-Pizlo, R. Wilensky and K. March, Effect of atherosclerosis on transmural convection and arterial ultrastructure, Arterio. Thromb. Vasc. Biol., 17 (1997), pp. 3365-3375.

[4] K. M. Balss, G. Llanos, G. Papandreou and C. A. Matyanoff, Quantitative spatial distribution of sirolimus and polymers in drug-eluting stents using confocal Raman microscopy, J. Biomed. Mater. Res., Part A, 85A(1) (2007), pp. 258-270.

[5] B. E. Bierer, P. S. Patilla, R. F. Standaert, L. A. Herzenberg, S. J. Burakoff, G. CRABTREe AND S. SChreiBer, Two distinct signal transmission pathways in T lymphocytes are inhibited by complexes formed between an immunophilin and either FK506 or rapamycin, Proc. Natl. Acad, Sci. USA, 87 (1990), pp. 9231-9235.

[6] P. Biscari, S Minisini, D. Pierotti, G. Verzini and P Zunino, Controlled release with finite dissolution rate, SIAM J. Appl. Math., 71(3) (2011), pp. 731-752.

[7] A. Borghi, E. Foa, R. Balossino, F. Migliavacca and G. Dubini, Modelling drug elution from stents: effects of reversible binding in the vascular wall and degradable polymeric matrix, Comput. Methods Biomech. Biomed. Eng., 11(4) (2008), pp. 367-377.

[8] T. Casalini, M. Masi and G. Perale, Drug eluting sutures: A model for in vivo estimations, Int. J. Pharm., 429 (2012), pp. 148-157.

[9] D. S. Cohen And T. Erneux, Controlled drug release asymptotics, SIAM J. Appl. Math., 58(4) (1998), pp. 1193-1204.

[10] S. Constantini, F. Maceri And G. VAiro, Un modelo del rilascio di farmaco in stent coronarici (in Italian), Proceedings of the XVII National Congress of Computational Mechanics GIMC 2008.

[11] S. CoOK, And S. Windecker, Coronary artery stenting, Cardiovascular Catheterization and Intervention, 2010, pp. 412-428.

[12] J. Crank, The Mathematics of Diffusion, Clarendon Press Oxford, 1975.

[13] C. D'Angelo, P. Zunino, A. Porpora, S. Morlacchi, F. Migliavacca, Model reduction stretegies enable computational analysis of controlled drug release from cardiovascular stents, SIAM J. Appl. Math., 71(6) (2011), pp. 2312-2333.

[14] M. C. Delfour, A. Garon and V. Longo, Modeling and design of coated stents to optimize the effect of the dose, SIAM J. Appl. Math., 65(3) (2005), pp. 858-881.

[15] L. Formaggia, S. Minisini and P. Zunino, Modeling polymeric controlled drug release and transport phenomena in the arterial tissue, Math. Mod. Meth. Appl. S., 20(10) (2010), pp. $1759-1786$.

[16] M. Grassi, G. Pontrelli, L. Teresi, G. Grassi, L. Comel, A. Ferluga and L. Galasso Novel design of drug delivery in stented arteries : a numerical comparative study, Math. Biosci. Eng., 6(3) (2009), pp. 493-508.

[17] T. Higuchi Rate of release of medicaments from ointment bases containing drugs in suspension, J. Pharm. Sci., 50 (1961), pp. 874-875.

[18] M. Horner, S. Joshi, V. Dhruva, S. Sett and S. F. C. Stewart A two-species drug delivery model is required to predict deposition from drug-eluting stents, Cardiovasc. Eng. Technol., 1(3) (2010), pp. 225-234.

[19] D. R. Hose, A. J. Narracott, B. Griffiths, S. Mahmood, J. Gunn, D. Sweeney and P. V. LAWFORD A thermal analogy for modeling drug elution from cardiovascular stents, Comput. Methods Biomech. Biomed. Eng., 7 (2004), pp. 257-264.

[20] C. W. Hwang, D. Wu and E. R. Edelman, Physiological transport forces govern drug distribution for stent based delivery, Circulation, 104(7) (2001), pp. 600-605.

[21] G. Karner and K. Perktold, Effect of endothelial injury and increased blood pressure on albumen accumulation in the arterial wall: a numerical study, J. Biomechs. 33 (6) (2000), pp. 709-715.

[22] W. Khan, S. Farah And A. J. Domb, Drug eluting stents: developments and current status, J. Controlled Release, 161(2) (2012), pp. 703-712.

[23] V. B. Kolachalama, A. R. Tzafriri, D. Y. Arifin and E. R. Edelman, Luminal flow patterns dictate arterial drug deposition in stent-based delivery,J. Controlled Release, 133 (2009) pp. 24-30. 
[24] V. B. Kolachalama, E. G. Levine and E. R. Edelman, Luminal flow amplifies stent-based drug-deposition in arterial bifurcations, PLoS ONE, 4(12) (2009), pp. 1-9.

[25] P. LibBy, Atherosclerosis: The New View, Scientific American, 286 (2007), pp. 29-37.

[26] D. Lloyde-Jones et Al., Heart Disease and Stroke Statistics-2010 Update: A Report From the American Heart Association, Circulation, 121 (2010), pp. e46-e215.

[27] A. D. Levin, M. Jonas, C-W Hwang and E. R. Edelman, Local and systemic drug competition in drug-eluting stent tissue deposition properties, J. Controlled Release, 109 (2005), $236-243$.

[28] M. A. Lovich and E. R. Edelman, Computational simulations of local vascular heparin deposition and distribution, Am J. Physiol. Heart Circ. Physiol., 271(5) (1996), H2014-H2024.

[29] A. Lusis, Atherosclerosis, Nature, 407 (2000), pp. 233-241.

[30] S. McGinty, S. McKee, R. M. Wadsworth and C. McCormick, Modelling drug-eluting stents, Math. Med. Biol., 28 (2011), pp. 1-29.

[31] R. Mongrain, I. Faik, R. L. Leask, J. Rodes-Cabau, E. Larose and O. F. Bertrand, Effects of diffusion coefficients and struts apposition using numerical simulations for drug eluting coronary stents,J Biomech. Eng., 129(5) (2007), pp. 733-742.

[32] C. Murray and A. Lopez, Alternative projections of mortality and disability by cause 19902020: Global Burder of Disease Study, The Lancet, 349(9064) (1997), pp. 1498-1504.

[33] G. Pontrelli and F. DE Monte, Mass diffusion through two-layer porous media: an application to the drug eluting stent, Int. J. Heat Mass Trans., 50 (2007), pp. 3658-3669.

[34] G. Pontrelli and F. De Monte, Modelling of mass dynamics in arterial drug-eluting stents, J. Porous Media 12(1) (2009), pp. 19-28.

[35] G. Pontrelli And F. DE Monte, A multi-layer porous wall model for coronary drug-eluting stents, Int. J. Heat Mass Trans., 53(1) (2010), pp. 3629-3637.

[36] S. Prabhu and S. Hossainy, Modeling of degradation and drug release from a biodegradable stent coating, J. Biomed. Mater. Res., Part A, 80A(3) (2007), pp. 732-741.

[37] F. Rossi, T. Casalini, E. Raffa, M. Masi and G. Perale, Bioresorbable Polymer Coated Drug Eluting Stent: A Model Study, Mol. Pharm., 9(7) (2012), pp. 1898-1910.

[38] R. S. Schwartz, E. Edelman, R. Virmani, A. Carter, J. F. Granada, G. L. Kaluza, N. A. F. Chronos, K. A. Robinson, R. Waksman, J. Weinberger, G. J. Wilson and R. L. Wilensky, Drug-Eluting Stents in Preclinical Studies. Updated Consensus Recommendations for preclinical Evaluation, Circulation: Cardiovascular Interventions, 1 (2008), pp. 143-1153.

[39] A. Seidlitz, S. Nagel, B. Semmling, N. Grabow, H. Martin, V. Senz, C. Harder, K. Sternberg, K-P. Schmitz, H. K. Kroemer and W Weitschies, Examination of drug release and distribution from drug-eluting stents with a vessel-simulating flow-through cell,Eur. J. Pharm. Biopharm., 78(1) (2011), pp. 36-48.

[40] J. Siepmann, K. Elkharraz, F. Siepmann and D. Klose, How autocatalysis accelerates drug release from PLGA-based microparticles: A quantatative treatment, Biomacromolecules, 6 (2005), pp. 2312-2319.

[41] J. Tambaca, M. Kosor, S. Canic and D. Paniagua, Mathematical modeling of vascular stents, SIAM J. Appl. Math., 70(6) (2010), pp. 1922-1952.

[42] A. R. Tzafriri, A. Groothuis, G. Sylvester Price and E. R. Edelman, Stent elution rate determines drug deposition and receptor-mediated effects, J. Controlled Release, 161 (2012), pp. 918-926.

[43] G. Vairo, M. Cioffi, R. Cottone, G. Dubini and F. Migliavacca, Drug release from coronary eluting stents: A multidomain approach, J. Biomech., 43 (2010), pp. 1580-1589.

[44] C. YAng And H. M. BuRT, Drug-eluting stents: factors governing local pharmacokinetics, Adv. Drug Delivery Rev., 58 (2006), pp. 402-411.

[45] H. Q. Zhao, D. Jayasinghe, S. Hossainy and L. B. Schwartz, A theoretical model to characterize the drug release behavior of drug-eluting stents with durable polymer matrix coating, J. Biomed. Mater. Res., Part A, 100A(1) (2012), pp. 120-124.

[46] W. Zhu, and T. Masaki, A. K. Cheung, And S. E. Kern, In-vitro release of rapamycin from a thermosensitive polymer for the inhibition of vascular smooth muscle cell proliferation, J. Bioequiv. Availab. , 1 (2009), pp. 3-12.

[47] P. Zunino, Multidimensional pharmacokinetic models applied to the design of drug-eluting stents, Cardiov. Eng.: Int J., 4(2) (2004), pp. 181-191.

[48] P. Zunino, C. D'Angelo, L. Petrini, C. Vergara, C. Capelli and F. Migliavacca, Numerical simulation of drug eluting coronary stents: Mechanics, fluid dynamics and drug release, Comput. Methods Appl. Mech. Engrg, 198 (2009), pp. 3633-3644. 